\title{
Analysis on The Curative Effect of Urinary Fistula Repair with Penile Flip Flap
}

\author{
Wang Xia ${ }^{1}$, Xie Shi-qing ${ }^{2}$, Liu Bo ${ }^{3}$ \\ 1.1st author: Wang Xia, Department of radiology, Yongchuan Hospital of ChongQing Medical University, 402160, \\ https://orcid.org/0000-0003-4029-6654 \\ 2.Co-1st anthor: Xie Shi-qing ,3rd medical college ,class 2018-4, HuBei University of Medicine, 442000, \\ https://orcid.org/ 0000-0003-1219-4853 \\ 3.Corresponding Author: Liu Bo, Department of Pediatric Surgery, Yongchuan Hospital of ChongQing Medical \\ University, 402160, e-mail: 1962806553@qq.com, https://orcid.org/0000-0002-9570-6561
}

Received:5 December 2019 Accepted: 10 December 2019 Published: 31 December 2019

Cite this Article Wang Xia, Xie Shi-qiang, Liu Bo: Analysis on The Curative Effect of Urinary Fistula Repair with Penile Flip Flap [J].Medical Research,2019.1(1): 18-22, http://dx.doi.org/10.6913/MRHK.201912_1(1).0003

Copyright (C) 2020 Creative Publishing Co., Limited. All rights reserved. Email:kycbshk@gmail.com.

\section{ABSTRACT}

Objective To investigate the effect of urinary fistula repair with penile flip flap after hypospadias correction in children.

Methods Anastomotic fistula and coronal groove fistula were repaired with penile flip flap,and the result were analyzed .

Results The correction rate of anastomotic fistula repair was significantly higher than that of coronal groove fistula group.

Discussion The penile flip flap is safe and reliable for the repair of anastomotic fistula and coronal groove fistula after hypospadias correction. The correction rate is high, especially in the repair of anastomotic fistula, related to local circulation and scar hyperplasia.

Keywords urinary fistula; repair; children

\section{INTRODUCTION}

Urinary fistula is the most common complication post-operatively of hypospadias repair. The incidence rate varies from literature to literature, ranging from $5 \%$ to $30 \%$, and the failure rate of urinary fistula repair is $20 \%$ to $40 \%{ }^{[1-5]}$. Many children with urinary fistula can not achieve the goal of healing after repeated repair, and families are physically and mentally exhausted. Therefore, increasing the cure rate of urinary fistula correction is the direction of surgeon. The common postoperative urinary fistula in hypospadias includes anastomotic fistula and coronal groove fistula. We used penile flip flap to repair the two urinary fistulas and analyzed the results.

\section{COMMON DATA}

From Jan. 2014 to Sept. 2019, 54 cases of urinary fistula repair postoperatively of hypospadias urethroplasty were performed, aged 3-13 years old(mean $5.5 \mathrm{y}$ ).

Table 1 Case number of anastomotic fistula /coronal groove fistula

\begin{tabular}{|l|l|l|}
\hline category & anastomotic fistulas & coronal groove fistulas \\
\hline Case number & 18 & 36 \\
\hline
\end{tabular}




\section{METHODS}

Hospitalization flow and perioperation step chart.(see Fig. 1)

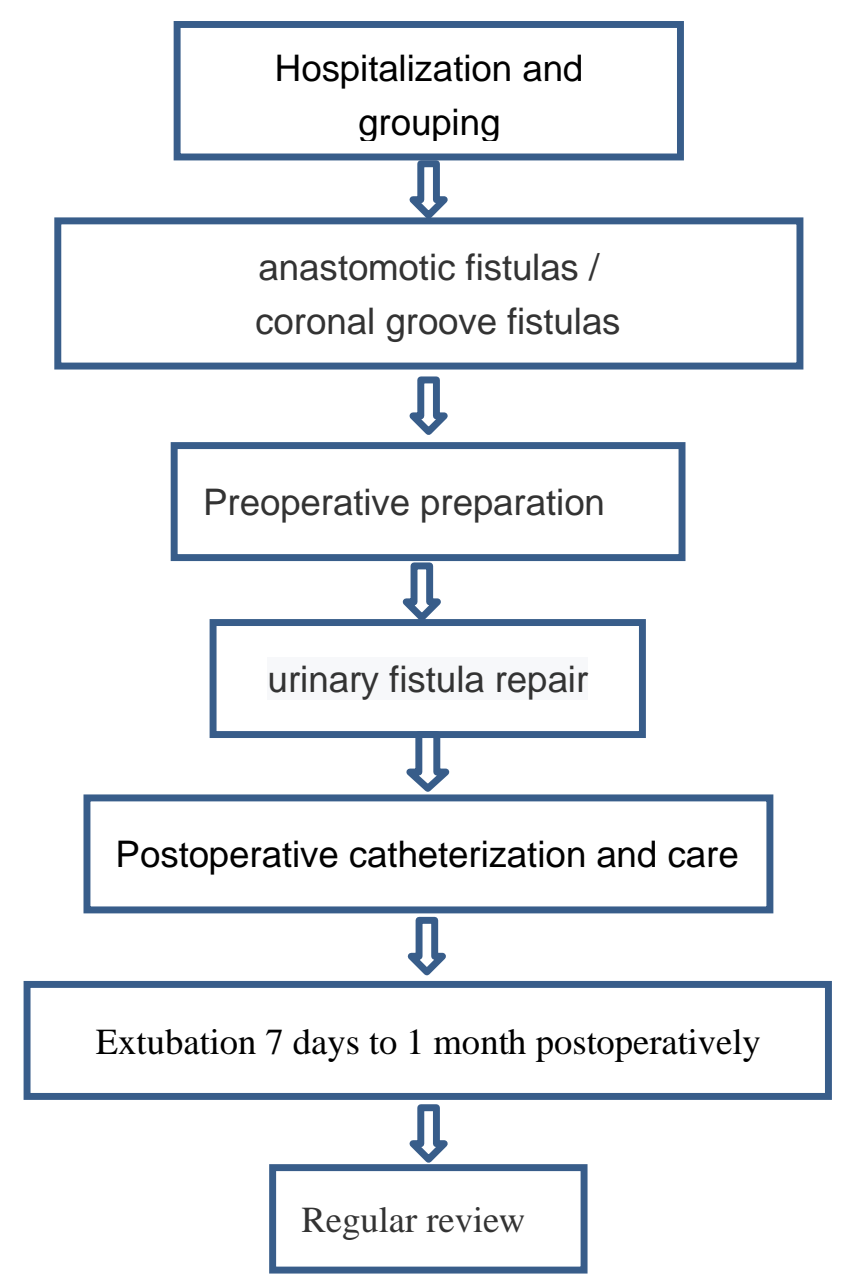

Fig 1 Hospitalization flow and perioperation step chart

\section{SURGERY STEPS}

Preoperative examination was completed for all children, and the operation was performed under tracheal intubation general anesthesia. Double-chamber urethral catheter was inserted before surgery。

Anastomotic urethral fistula: the fistula is mostly located in the proximal or middle segment of the penile ventral. Firstly, the subcutaneous tissue around the fistula should be trimmed, the scar tissue cut off, the fistula exposed and the urethra protected .The distal penile/scrotal midline middle-thick flap was revised via an inverted U-shaped incision and turned over to cover the fistula with $6 / 0$ absorbable continuously suture. And then the flip flap was capped with distal subcutaneous fascia with intermittent suture. In the end Prune the skin and suture the incision continuously.

Coronary groove fistula: The skin scar around the fistula were trimmed first, the urethra protected. The fistula was closed, under the oblique $45^{\circ}$ rectangular incision, with the proximal lateral phallic flip flap, continuous suture and intermittent reinforced. Another incision was cut on the other proximal side of the fistula, the subcutaneous fascia turned-over and covered the flap. The skin incision was closed with continuous and intermittent suture.

\section{RESULT}

The 54 children were grouped according to the fistula, and undergone the repair surgery In accordance with the operation steps above. Preoperative and postoperative treatment process were performed in accordance 
Medical Research ISSN 2664-0333 Volume 1 No.1 PP.18-22 http://dx.doi.org/10.6913/MRHK.201912_1(1).0003

Wang Xia, Xie Shi-qiang, Liu Bo: Analysis on The Curative Effect of Urinary Fistula Repair with Penile Flip Flap

with the procedure. The postoperative catheter indwelling duration is basically 14 days( 7 days- 1 month) . Anastomotic fistula is located at the proximal part of the penis, and the local scar is light and few. The subcutaneous tissue around the fistula is healthy and distinct. The blood supply of pedicled flap is well. The coronal groove fistula is located at the distal end of the penis with poor local tissue hierarchy, heavy scar and poor flap condition. After repair, some cases of skin flap necrosis and then scar healing, continued indwelling catheter is conducive to local scar healing. So, the indwelling catheter should be maintained for up to 1 month, and the distal urathra should be dilatated regularly after extubation. Urethral dilatation can be instructed to parents after health education. Regular follow-up adjustment.

In this study, the maximal frequency of repair was: anastomotic fistula 3 times, while coronal groove fistula 8 times.

Table 2. Frequency of urinary fistula repair

\begin{tabular}{|c|c|c|c|c|c|c|c|c|}
\hline Frequency & 1 & 2 & 3 & 4 & 5 & 6 & 7 & 8 \\
\hline cases & 36 & 13 & 2 & 1 & 1 & 0 & 0 & \\
\hline
\end{tabular}

Table 3 . The success ratio of fistula repair in two groups

\begin{tabular}{|c|c|c|}
\hline & anastomotic fistula group & coronal groove fistula group \\
\hline achievement ratio of operation & $17 / 18(94.4 \%)$ & $23 / 36(63.8 \%)$ \\
\hline
\end{tabular}

The comparison of fistula repair success ratio in two groups was statistically significant (SPSS 19.0) $(\mathrm{P}<0.05)$.There was a significant difference in the healing rate between anastomotic fistula and coronal groove fistula repair. The result was related to the severity of local anatomical scar and distribution of blood supply.

\section{CONCLUSION}

Urinary fistula is the most common complication post-operative of hypospadias urethroplasty, and the cause include: distal urethral stricture of new urethra, urethral outer-opening stricture, urethral diverticulum , lack of support tissue in the new urethra, poor blood supply in the operative flap, necrosis or infection of the flap and poor urine drainage, etc ${ }^{[7]}$.

Most scholars believe that the surgical timing of urethral fistula repair should be performed 6 months after the last operation and preferably 1 year later, when the local scar tissue ,fixed, will not develop, and the tissue around will return to health without inflammation. At the same time, there should be no obvious signs of urine infection preoperatively.At least 3 months after the last surgery, the scar around the urine fistula turned softened and limited, so that a new blood supply could be established.

At the same time, the distal urethral stricture should also be estimated, and urethral stenosis should be treated. Otherwise, morbility of fistula repair will increase obviously ${ }^{[8]}$.

To improve the cure rate of urinary fistula repair is the key to improve hypospadias family medicalsatisfaction and physical and mental health .

Urinary fistula after hypospadias surgery included anastomotic fistula and coronal groove fistula.The surgery of urinary fistula included direct repair, flap repair and urinary reconstruction. Flap repair is most adopted, with less trauma, higher success rate or lower reoperative rate, without causing urethral stricture

For anastomotic fistulas and coronal groove fistulas, we used penile proximal over- flip flap repair technique. The proximal penile and scrotal midline flap for the anastomotic fistula repair had no surgical scar tissue, with good blood supply. The scar around anastomotic fistula was lighter than the coronal groove fistula, and 
Medical Research ISSN 2664-0333 Volume 1 No.1 PP.18-22 http://dx.doi.org/10.6913/MRHK.201912_1(1).0003

Wang Xia, Xie Shi-qiang, Liu Bo: Analysis on The Curative Effect of Urinary Fistula Repair with Penile Flip Flap

subcutaneous tissue still existed around the fistula. The penis- scrotal junction and the scrotal midlinel flap are axial island flaps with the anterior and posterior scrotal arteries and their anastomotic branches as the vascular pedicles ${ }^{[6]}$. After the fistula freed, the inverted $\mathrm{u}$-shaped proximal rectangular flap of peri-fistula was reversed and the fistula was continuous varus suture, with small tension and good blood supply. Then covered with a healthy peripherally pedicled fascia, the skin incision was reshaped. It is beneficial to repair and heal the fistula.

In the ventral side of the coronal groove of the penis, the coronal groove fistula scar contracture is obvious, because the coronal groove of the penis itself is a natural scar with few adjacent healthy flaps and subcutaneous fascia. There is no subcutaneous tissue between the skin scar and urethral fistula, and the incision and release around the fistula can easily damage the urethra and cause the enlarged fistula, making repair more difficult.

Therefore, it is impossible to adopt the proximal flap of the fistula. The completely vertical lateral flap does not meet the optimal distribution of blood supply, but the reversed- "Y" rectangular flap that avoids the central axis of the urethra ,not only preserves the excellent blood supply and collateral circulation of the proximal end, but also avoids the damage to the urethra. At the same time, the free coronary groove fistula was as far away from the fistula as possible, free to healthy tissue outside the urethral tube around the fistula, fully cut out the remaining skin scar on the fistula, and then closed the fistula with the inverted flap varus, and the healthy fascia on the other side was flipped and covered with a layer. Skin incisions were cut and then sutured.

We used double - chamber super - slip urinary catheters for hypospadias or urethroplasty in our center. Normal saline lubrication is convenient for implantation, and 5-7 days after the operation, urinary tube and wound adhesion is separated, which is easy to be removed, causing little damage to the urethra, and there is no damage to the early tube removal, or difficult extubation damage to the urethra. The operation quality is guaranteed, and the nursing is simple. Reduce expenses and average hospital stay. According to the literature, there was no significant difference in the effectiveness of the single-chamber silicone catheter sewn into the penis head with a traction line, or the double-chamber catheter of the corresponding size.

Through the comparative study of this group, the penile flap is safe and reliable for the repair of anastomotic fistula and coronal groove fistula after hypospadias, with a high success rate, especially in the repair of anastomotic fistula has a very obvious advantage. Due to the good local anatomical conditions, the healing rate of stage 1 repair operation is high, and the total number of repair operations is also low. However, due to poor local conditions (scar, blood supply, etc.) and poor overall healing rate in stage 1 , coronal groove fistula often requires multiple surgeries.

"The more sewing repair, the more leakage", looking forward to more improved surgical skills and even tissue engineering materials to improve the success rate of the repair of urinary fistula, improve the experience of medical treatment for children, and improve the physical and mental health of children and their families.

\section{REFERENCES}

1 liu bo, wang xia, liu xing, Chen taizhong. Clinical study of infant modified Snodgrass hypospadias correction [J].Chinese journal of general practitioners, 2018, 17 (8) : 629-631

2 Liu bo, Chen taizhong, li guangliang et al. Modified Snodgrass repair for 62 cases of hypospadias in children [J].Journal of clinical pediatric surgery, 2013,12 (1) : 72-73

3 Liu bo, wang xia, Chen yong, et al. Analysis of recurrent urinary tract infection postoperative of hypospadias [J]. Journal of clinical pediatric surgery, 2015 (2) : 113-116,129 
Medical Research ISSN 2664-0333 Volume 1 No.1 PP.18-22 http://dx.doi.org/10.6913/MRHK.201912_1(1).0003

Wang Xia, Xie Shi-qiang, Liu Bo: Analysis on The Curative Effect of Urinary Fistula Repair with Penile Flip Flap

4 Liu bo, wang xia, Chen taizhong et al. Analysis of Y-shaped scrotal flap for repair of severe hypospadias in children [J].Laboratory medicine and clinical practice, 2014 (11) : 1521-1522,1525

5 Liu bo, wang xia, Chen taizhong et al. Clinical study on the prevention of hypospadias infection under blood scab with soluble hemostatic gauze in children [J]. Laboratory medicine and clinical practice, 2014 (12) : 1674-1675

6 Duan chenwang, Huang xunlei, Chen bin,etc. Repair of hypospadias using scrotal septum vascular pedicle flap with two wing-like split-thickness skin graft $[\mathrm{J}]$.Chinese Journal of Reparative and Reconstructive Surgery, 2009,23 (3):316-318

7 Sheng X, Xu D, Wu Y, et al. The risk factors of Urethrocutaneous fistula after hypospadias surgery in the youth population. BMC Urol 2018;18:64. Available at https://bmcurol.biomedcentral.com/articles/10.1186/s12894-018-036 6-Z. [accessed May 27, 2019]

8 Cimador M, Castagnetti M, De Grazia E, et al. Risks and relevance of preputial reconstruction in hypospadia repair. Pediatr Med Chir 2019;25:269-72. Available at http://www.ncbi.nlm.nih.gov/pubmed/15070270. [accessed July 17] 